\title{
ORAL SESSION
}

\section{ORAL SESSION 4B HEART AND HAEMODYNAMICS}

\section{OP.4B.01 LEFT VENTRICULAR MECHANICS IN PATIENTS WITH MASKED HYPERTENSION}

M. Tadic ${ }^{1}$, C. Cuspidi ${ }^{2}$, V. Vukomanovic ${ }^{1}$, B. Pencic ${ }^{1}$, V. Kocijancic ${ }^{1}$, V. Celic ${ }^{1}$. ${ }^{1}$ University Hospital Dragisa Misovic, Belgrade, SERBIA, ${ }^{2}$ University of MilanBicocca and Istituto Auxologico Italiano, Milan, ITALY

Objective: We sought to investigate left ventricular (LV) mechanics in subjects with masked hypertension $(\mathrm{MH})$, normotensive and sustained hypertensive patients.

Design and method: This cross-sectional study included 209 untreated subjects who underwent 24-hour ambulatory BP monitoring and complete two-dimensional echocardiographic examination (2DE) including multilayer strain analysis. $\mathrm{MH}$ was diagnosed if clinic blood pressure (BP) was normal $(<140 / 90 \mathrm{mmHg})$ and 24-hour BP was increased ( $>=130 / 80 \mathrm{mmHg}$ ).

Results: According to criteria provided in the Methods, the study sample included 62 normotensive subjects (30\%), $64 \mathrm{MH}(30 \%)$ and 83 sustained hypertensives (40\%). 2DE LV longitudinal strain gradually and significantly decreased from normotensive controls across $\mathrm{MH}$ individuals to sustained hypertensive patients $(-21.3 \pm 2.1$ vs. $-19.1 \pm 2$ vs. $-17.4 \pm 1.8 \%, \mathrm{p}<0.001)$. The same is valid for $2 \mathrm{DE}$ LV circumferential strain $(-21.9 \pm 2.9$ vs. $-20 \pm 2.5$ vs. $-18.4 \pm 2.2 \%, p<0.001)$ $2 \mathrm{DE}$ radial strain was not different between groups. $2 \mathrm{DE}$ LV longitudinal endocardial and mid-myocardial layer strains progressively decreased from normotensive control to sustained hypertensive individuals $(-26.4 \pm 4.6$ vs. $-23.8 \pm 3.8$ vs. -21 $\pm 3.6 \%, \mathrm{p}<0.001$ for endocardial strain; and $-21 \pm 3.2$ vs. $-18.8 \pm 2.8$ vs. $-16.8 \pm$ $2.5 \%, \mathrm{p}<0.001$ for mid-myocardial strain). Similar results were obtained for $2 \mathrm{DE}$ LV circumferential endocardial and mid-myocardial layer strains $(-28.6 \pm 5$ vs. $-25.4 \pm 4.4$ vs. $-23.2 \pm 4 \%, \mathrm{p}<0.001$ for endocardial strain; and $-21.5 \pm 2.8$ vs. $-19.4 \pm 2.4$ vs. $-18.2 \pm 2.2 \%, p<0.001$ for mid-myocardial strain). Longitudinal and circumferential epicardial layer strains were decreased in sustained hypertensive patients than in normotensive controls. 24-hour systolic BP was associated with peak 2DE LV longitudinal $(\beta=-0.442, p<0.001)$, endocardial strain $(\beta$ $=-0.394, \mathrm{p}<0.001)$, mid-myocardial strain $(\beta=-0.426, \mathrm{p}<0.001)$ and $2 \mathrm{DE}$ circumferential endocardial strain $(\beta=-0.364, p<0.001)$ in the whole study population independent of $\mathrm{LV}$ mass index and $\mathrm{E} / \mathrm{e}$ ' ratio.

Conclusions: $\mathrm{MH}$ significantly influences LV deformation assessed by $2 \mathrm{DE}$ traditional strain and 2DE multilayer strain. 24-hour systolic BP is associated with LV deformation evaluated with comprehensive $2 \mathrm{DE}$ strain analysis independent of LV structure and diastolic function.

\section{OP.4B.02 NON INVASIVE EVALUATION OF END SYSTOLIC LEFT VENTRICULAR ELASTANCE ACCORDING TO PRESSURE-VOLUME CURVE MODELING DURING EJECTION IN ARTERIAL HYPERTENSION}

B. Bonnet ${ }^{1,2}$, F. Jourdan ${ }^{2}$, G. du Cailar ${ }^{1}$, P. Fesler ${ }^{1} .{ }^{1}$ Department of Internal Medicine, CHU, Montpellier, FRANCE, ${ }^{2}$ Laboratoire de Mécanique et Génie Civil, Montpellier, FRANCE

Objective: End systolic left ventricular (LV) elastance (Ees) has been previously calculated and validated invasively using LV pressure-volume (P-V) loop. Non invasive studies have been proposed, but clinical application remains complex. The aim of the present study was to 1) estimate Ees according to modeling of LV P-V curve during ejection ("Ejection PV Curve" method) and validate our method with existing published LV P-V loop data; 2) test clinical applicability to detect non invasively a difference in Ees between normotensive and hypertensive subjects.

Design and method: Based on P-V curve and a linear relationship between LV elastance and time during ejection, we used a non linear least square method to fit the systolic pressure curve. We then computed slope and intercept of time varying elastance, and calculated Ees as LV elastance at the end of ejection. As a validation, $22 \mathrm{P}-\mathrm{V}$ loops obtained from previous invasive studies were digitized and analyzed with our method. To test clinical applicability, P-V curve was obtained from 33 hypertensive and 32 normotensive subjects, using carotid tonometry and real time $3 \mathrm{D}$ echocardiography.
Results: A good univariate relationship $\left(r^{2}=0.92, p<0.005\right)$ and a good limit of agreement were found between previous invasive calculation of Ees and our new proposed "Ejection P-V Curve" method. In addition, the clinical reproductibility of our method was similar to that of another non invasive method proposed by Chen et al. In hypertensive patients, the increase in arterial elastance (Ea) was compensated by an increase in Ees without change in $\mathrm{Ea} / \mathrm{Ees}$ (see Figure).
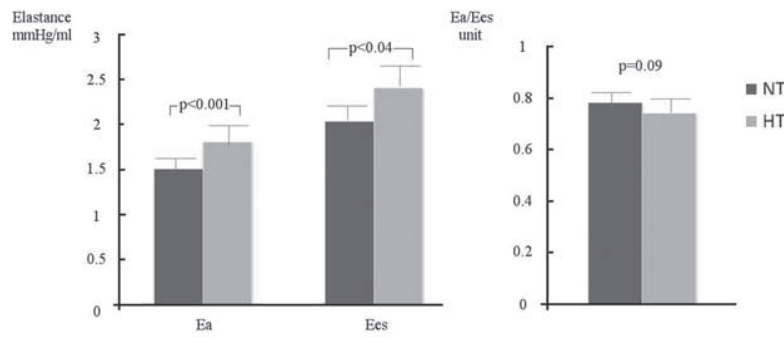

Conclusions: Ees can be estimated non invasively from modeling of P-V curve during ejection. This approach was found to be reproducible and sensitive enough to detect an expected difference in LV contractility in hypertensive patients. Due to its non invasive nature, this methodology may have clinical implications in various disease states.

OP.4B.03

CIRCULATING ALDOSTERONE LEVELS ARE
ASSOCIATED WITH CONCENTRIC LEFT
VENTRICULAR GEOMETRY IN ESSENTIAL
HYPERTENSIVE PATIENTS

G. Mule', E. Nardi, L. Guarino, M. Morreale, D. Altieri, G. Geraci, M. Guarneri, V. Cacciatore, G. Cerasola, S. Cottone. Unit of Nephrology and Hypertension - ESH excellence Centre - DIBIMIS - University of Palermo, PALERMO, ITALY

Objective: Sound evidence indicates that aldosterone has a fundamental role in determining functional and structural changes in the heart. Moreover, it has been observed that high plasma aldosterone concentration (PAC) is related to the development of congestive heart failure and to cardiovascular mortality. However, previous studies on the association between circulating aldosterone levels and left ventricular (LV) mass (LVM) and LV geometry, in subjects without primary aldosteronism yielded conflicting results. The aim of our study was to evaluate in patients with essential hypertension the relationships of PAC with LV mass and geometry, and to asses the influence of gender on these relationships.

Design and method: A total of 478 subjects (men: $63 \%$; mean age $44 \pm 12$ years) with untreated essential hypertension were enrolled.

The measurements included 24-h blood pressure (BP) readings, plasma renin activity (PRA) and PAC, obtained by radioimmunoassay and an echocardiogram.

For its skewed distribution PAC was log transformed and expressed as median value and interquartile range.

Results: PAC was significantly lower $(\mathrm{p}<0.01)$ in subjects with normal LVM indexed for body surface area $(\mathrm{BSA})(\mathrm{n}=272)[7.5(5.3-12.4)]$, as compared to those with concentric remodeling $(\mathrm{n}=61)[10.2$ [7.95-14.5)], to the patients with eccentric LV hypertrophy $(\mathrm{LVH})(\mathrm{n}=90)[9.8(6.9-12.9)]$, and to the subjects with concentric LVH $(\mathrm{n}=61)[11.3(7.7-16.6 \mathrm{ng} / \mathrm{ml})]$. Significant correlations of $\mathrm{Log}$ (PAC) with LVM, either indexed for BSA $(r=0.20 ; \mathrm{p}<0.0001)$, or for height 2.7 $(\mathrm{r}=0.21 ; \mathrm{p}<0.0001)$ and with relative wall thickness (RWT) $(\mathrm{r}=0.18$; $\mathrm{p}<0.0001$ ) were found. These correlations were similar in men and in women and remained statistically significant in multiple regression analyses, even after adjustment for potential confounding factors (all $\mathrm{p}<0.01$ ).

Conclusions: Our results seem to suggest that in essential hypertensive patients circulating aldosterone levels are independently associated with concentric LV geometry, without gender-related differences.

\section{OP.4B.04 \\ LONGITUDINAL CHANGES IN LEFT VENTRICULAR STRUCTURE AND DIASTOLIC FUNCTION IN RELATION TO ARTERIAL PROPERTIES IN A GENERAL POPULATION}

N. Cauwenberghs ${ }^{1}$, J. Knez ${ }^{1}$, J. D'hooge ${ }^{2}$, L. Thijs ${ }^{1}$, W.Y. Yang ${ }^{1}$, F. Haddad ${ }^{3}$, J.A. Staessen ${ }^{1}$, T. Kuznetsova ${ }^{1} .{ }^{1} K U$ Leuven - Research Unit of Hypertension and 